\title{
TELEMEDICINE DURING THE COVID-19 PANDEMIC: EXPERIENCE FROM AN ITALIAN OUTPATIENT CLINIC FOR MOVEMENT DISORDERS
}

\author{
Susanne Buechner, Andrea La Licata, Dario Seppi, Francesco Teatini \\ Department of Neurology/Stroke Unit, Bolzano Hospital, Bolzano/Bozen, Italy
}

\begin{abstract}
Patients with chronic diseases such as Parkinson's disease need periodic medical examinations. During the COVID-19 pandemic non-emergent medical visits were impossible. To ensure continuity of health care, telemedicine has been demonstrated to be a potential and useful tool.
\end{abstract}

Key words: COVID-19, Movement Disorders, Parkinson's Disease, Telemedicine.

Coronavirus disease 2019 (COVID-19), caused by the severe acute respiratory syndrome coronavirus 2 (SARS-CoV-2), has rapidly become a world pandemic [1]. The fear of coronavirus contagion has led to selfisolation and social distancing. In Italy, a hotspot of the pandemic, the COVID-19 infection spread within a few weeks from the initial foci (i.e. Codogno, a small town close to Milan, Lombardy) to the rest of the country [2]. In order to slow down the wave of infections, drastic governmental measures for containment of transmission were enacted. On March 8, 2020 a legal decree was issued, asking people of 16 North Italian provinces to stay indoors unless they need to buy food or medicine. These severe limitations to mobility were subsequently widened to the whole of Italy. Non-emergent surgical procedures and medical outpatient visits were also cancelled. However, patients with chronic diseases, such as Parkinson's disease (PD), need routine examinations for physical assessment and medication adjustments.

In this setting of COVID-19, telemedicine (TM) has been promoted and used to triage or monitor COVID-19 patients, reducing the risk of transmission, especially in the United Kingdom and the United States of America [3]. TM has already been suggested as a response to public health emergencies [4]. The functional definition of $\mathrm{TM}$ is medicine delivered over distance, whereby the communication can be in real-time (i.e. synchronous) or not (i.e. asynchronous) [5]. TM has been around in some form for many centuries and some authors relate the first use of TM to be when ancient civilizations sent smoke signals to warn other clans of a contagious illness outbreak [6]. Of course, TM has changed during the centuries, especially in the last decades due to significant and rapid improvement in the underlying enabling digital

Correspondence to: Susanne Buechner, M.D.

Department of Neurology/Stroke Unit, General Hospital of Bolzano

Lorenz Boehler Street, 539100 Bolzano/Bozen, Italy

Phone: +390471 907589

E-mail: susanne.buechner@sabes.it

Received May $18^{\text {th }}, 2020$ / Accepted June $21^{\text {st }}, 2020$ technologies. There are many different types of TM, and phone calls or video conferencing replacing in-person outpatient visits are only one kind of TM [5]. TM has been already used successfully for many different fields, for example in asthma care over distance [7] or for diabetic retinopathy screening [8]; the validity of TM to assess PD patients has been also well documented [9].

Our Movement Disorders Outpatient Clinic is located in the Hospital of Bolzano, which belongs to the Autonomous Province of South Tyrol in Northern Italy. Our clinic provides, for more than 1000 patients, access to three neurologists specialized in diagnosing and treating movement disorders for assessment and follow-up. Our team includes three nurses, one of which is a PD nurse specialist. Our clinic also provides opportunities for patients to participate in research studies related to movement disorders, characterized mainly by collaborative projects with other movement disorder outpatient clinics.

In order to guarantee continuity of medical care for our established patients during the COVID-19 pandemic, our ambulatory in-person appointments were immediately converted to phone calls. All patients who had scheduled an in-person visit for March or April 2020 were called by one of the three neurologists of our Movement Disorders Outpatient Clinic. In fact, no preselection of patients' suitability for TM had been performed; in case of patients with cognitive impairment or reduced ability to use technology, we directly contacted their caregivers.

We have made some interesting observations using TM. From March 10 until April 27, 2020 we called 150 patients from our Movement Disorders Outpatient Clinic. No phone call was refused, and all patients wished for a telemedical contact rather than a face-toface consultation in order to avoid contact with potentially infected persons. In total, 165 telephone-calls were made, as some patients had to be called two (11 patients) or three times ( 2 patients). The mean age of the patients was 74 years (range: 45-96); 70 were females, 80 were males. Most patients (115) had the diagnosis of 
idiopathic PD or Parkinsonism, 18 patients suffered from essential tremor or other kinds of tremor, 14 patients had restless-legs syndrome, while the remaining 3 patients were affected with rare movement disorders (2 patients with Huntington disease, 1 patient with stiff person syndrome) (Table 1).

In nearly two thirds of the patients, clinical-therapeutic problems could be resolved during the telephone-call, and therefore the following out-patient visit could be scheduled for autumn. In 58 patients the phone call was not conclusive, and therefore an in-person visit was necessary as soon as possible (Figure1). The mean age of these 58 patients was 72 years and most of them, namely 52 patients, suffered from PD or Parkinsonism, 34 of which for more than 5 years. In 24 patients from this group, the current therapy was modified during the telephone-call; 35 patients were on medication with two or more Anti-Parkinson drugs. Thus, these aspects (severe movement disorder disease, polymedication, advanced age) associated to other comorbidities (e.g. arterial hypertension or diabetes mellitus) influenced the need for an in-person encounter.

In summary, despite one third of the 150 contacted patients of our Movement Disorders Outpatient Clinic needing an in-person visit, for the majority the phone calls were successful and could substitute the scheduled appointments. Of course, a phone-conversation is not the same as an in-person encounter and cannot replace an outpatient clinic, however, many of our patients seemed quite satisfied with the contact by telephone. This TM experience was also associated with satisfaction among our staff, permitting uninterrupted care of our patients, reducing the risk of SARS-CoV-2 transmission to other patients and ourselves and saving personal protective equipment. Finally, phone consultation worked without particular difficulties, and all patients were able to respond more or less immediately to the phone calls due to the lockdown introduced by the Italian government.

Certainly, we have identified the need to improve our telehealth technology in the near future, using video-conferencing instead of "simple" telephone-calls according to the practical guidance offered by the International Parkinson and Movement Disorder Society [10] and recommended by other movement disorders specialists [11], as video consultations would give us also the possibility to see the patient and to assess clinical features. However, for this pandemic there was no time for adoption of video conferencing solutions, mainly due to technical issues, such as installing a dedicated video software or web cameras on our and patients' computers. Unfortunately, we were also unprepared for video consultations because Italy does not include TM in the essential levels of care granted to all citizens within the National Health Service [3].

However, we should think about integration of TM within our health care system, guaranteeing, of course, a

Table 1 Demographic and clinical characteristics of the contacted patients

\begin{tabular}{lll}
\hline $\begin{array}{l}\text { Total number } \\
\text { and sex of pts. }\end{array}$ & $\begin{array}{l}\text { Mean age of pts. } \\
\text { (years) }\end{array}$ & $\begin{array}{l}\text { Diagnosis with disease duration }(>5 \text { years) } \\
\text { and PD polymedication }(\geq 2 \text { drugs) (number of pts.) }\end{array}$ \\
\hline 150 & 74 (range 45-96) & PD/Parkinsonism: $115 ;>5$ years: $76 ; \geq 2$ drugs: 67 \\
70 females & 75 (range 45-96) & ET/tremor: $18 ;>5$ years: 11 \\
80 males & 73 (range 45-93) & RLS: $14 ;>5$ years: 9 \\
& & HD: $2 ;>5$ years: 1 \\
& & SPS: $1 ;>5$ years: 1 \\
\hline
\end{tabular}

Abbreviations: pts.: patients; PD: Parkinson's disease; ET: essential tremor;

RLS: restless-legs syndrome; HD: Huntington disease; SPS: stiff person syndrome

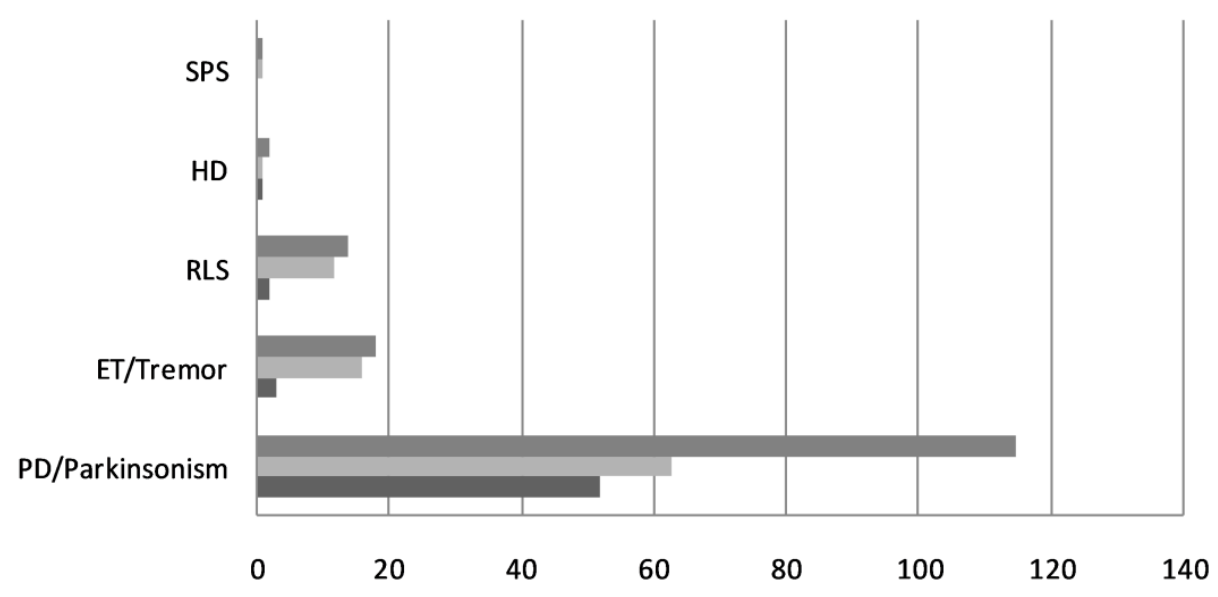

$\square \mathrm{N}^{\circ}$ of patients called $\quad \mathrm{N}^{\circ}$ of conclusive phone-calls $\quad \mathrm{N}^{\circ}$ of inconclusive phone-calls

Fig. 1 Outcome of phone-calls during COVID-19 pandemic

Abbreviations: SPS: stiff person syndrome, HD: Huntington disease,

RLS: restless-legs syndrome, ET: essential tremor, PD: Parkinson's disease 
high-quality standard. Furthermore, after the current COVID-19 pandemic emergency TM might be considered a useful supplementary tool for our outpatient clinic, especially for established patients who are fragile, who have significant difficulties of moving around and who need to be accompanied by a caregiver. In these cases, the utilization of TM would save travelcosts and caregiver work-days, providing a positive effect also on the economy and on the environment. Of course, implementing TM takes time and does not happen overnight. TM is highly complex and full of challenges, and its integration requires precise guidelines.

\section{References}

1. Lu R, Zhao X, Li J, Niu P, Yang B, Wu H, et al. Genomic characterization and epidemiology of 2019 novel coronavirus: implications for virus origins and receptor binding. Lancet 2020; 395:565-574. doi: 10.1016/s0140-6736(20)30251-8

2. Gatto M, Bertuzzo E, Mari L, Miccoli S, Carraro L, Casagrandi $\mathrm{R}$, et al. Spread and dynamics of the COVID-19 epidemic in Italy: Effects of emergency containment measures. PNAS 2020; 117:10484-10491. doi: 10.1073/pnas.2004978117

3. Ohannessian R, Duong TA, Odone A. Global Telemedicine implementation and integration within health systems to fight the COVID-19 pandemic: A call to action. JMIR Public Health Surveill. 2020; 6(2):e18810. doi: 10.2196/18810

4. Lurie N, Carr BG. The role of Telehealth in the medical response to disasters. JAMA Intern Med 2018; 178:745-746. doi: 10.1001/ jamainternmed.2018.1314

5. Waller M, Stotler C. Telemedicine: a Primer. Curr Allergy Asthma Rep 2018; 18(10):54. doi: 10.1007/s11882-018-0808-4

6. Hurst EJ. Evolutions in telemedicine: from smoke signals to mobile health solutions. J Hosp Librariansh 2016; 16:174-185. doi: 10.1080/15323269.2016.1150750
Adequate technical equipment, including modern devices and a secure, high-speed Internet connection, is needed, which must be available for everybody, and easy to use also by elderly patients, unless relatives are on hand to help. Other challenges of TM include legal aspects, payment, credentialing, ethical considerations and staff training. In conclusion, although the presence of the COVID-19 pandemic is a dramatic event, it is also an opportunity for us to implement TM and to change something in long established processes and routines in our medical health care system.

7. Portnoy JM, Waller M, De Lurgio S, Dinakar C. Telemedicine is as effective as in-person-visits for patients with asthma. Ann Allergy Asthma Immunol 2016; 117:241-245. doi: 10.1016/j.anai.2016. 07.012

8. Rachapelle S, Legood R, Alavi Y, Lindfield R, Sharma T, Kuper $\mathrm{H}$, et al. The cost-utility of telemedicine to screen for diabetic retinopathy in India. Ophthalmology 2013; 120:566-573. doi: 10.1016/j.anai.2016.07.012

9. Ben-Pazi H, Browne P, Chan P, Cubo E, Guttman E, Hassan A, et al. The promise of telemedicine for movement disorders: an interdisciplinary approach. Curr Neurol Neurosci Rep 2018; 18(5):26. doi: 10.1007/s11910-018-0834-6

10. International Parkinson and Movement Disorder Society. Telemedicine Study Group. Telemedicine in Your Movement Disorders Practice. https://www.movementdisorders.org/MDS/About/ Committees--Other-Groups/Telemedicine-in-Your-MovementDisorders-Practice-A-Step-by-Step-Guide.htm

11. Papa SM, Brundin P, Fung VSC, Kang UJ, Burn DJ, Colosimo C, et al. Impact of the COVID-19 Pandemic on Parkinson's Disease and Movement Disorders. Mov Disord Clin Pract 2020; 7 : 357-360. doi: 10.1002/mds. 28067 